\title{
Escalamiento de resultados de flotación de minerales a través de la función $\mathrm{DTR}^{\left({ }^{\circ}\right.}$
}

\author{
L. Magne $\left(^{(*)}\right.$, J. Jofré( ${ }^{(*)}$, J. Barría ${ }^{(* *)}$ y J. Menacho ${ }^{(* *)}$
}

\begin{abstract}
Resumen Se presenta una revisión de los principales modelos de distribución de tiempos de residencia, DTR, utilizables en flotación de minerales: modelos ideales de mezcla perfecta y flujo pistón, modelo de $N$ reactores perfectos en serie y modelo combinado general de varias etapas. El que se utiliza más frecuentemente es el de $N$ reactores imperfectos en serie, en el cual se atribuye una interpretación física invariante a los desplazamientos en tiempo e intensidad de la función DTR, pudiendo llevar a conclusiones erróneas respecto al régimen de mezcla y al cálculo de recuperación por flotación en planta. En este artículo se integra analíticamente la ecuación para escalar resultados de sistemas semicontinuos a planta industrial a través de la función DTR, empleando el modelo de mezcla imperfecta, y los modelos de García-Zúñiga y Klimpel para recuperación. Así, se logra una predicción más exacta del comportamiento de sistemas industriales a partir de información a menor escala, comparado con otros métodos.
\end{abstract}

Palabras clave: Distribución de tiempos de residencia. Flotación. Escalamiento de recuperación en flotación.

\section{Results of flotation scale-up from RTD function}

\begin{abstract}
A review on the main residence time distribution (RTD) models currently applied to flotation is presented. Perfect mix, plug flow, $N$ perfect mix reactor in series and the general combined model are included. In flotation diagnosis the most required model is the $N$ non perfect mix reactor in series (NPRS). As an invariant physical meaning is usually assigned to the different shift in time and intensity of the peaks, wrong conclusion may arise with regard to the real mix state and correct scaling-up of the flotation recovery. In the present paper the above situation is overcome by integrating semibatch results in an analytical way, with the assistance of the García-Zúñiga and Klimpel equations for the recovery and the NPRS model for the mix state. Highly accurate predictions at industrial scale are obtained as compared to other methods.
\end{abstract}

Keywords: Residence time distribution. Flotation. Recovery scale-up in flotation.

\section{INTRODUCCIÓN}

La función distribución de tiempos de residencia, DTR, de un fluido que circula en estado estacionario a través de un reactor continuo, representa la distribución estadística de tiempos de permanencia de los diferentes elementos del fluido en el reac-

(•) Trabajo recibido el día 24 de marzo de 1994.

(*) Dpto. de Ingeniería Metalúrgica. Facultad de Ingeniería. Universidad de Santiago de Chile. Casilla 10233. Santiago (Chile)

(**)Centro de Investigación Minera y Metalúrgica (CIMM). Casilla 170. Correo 10. Santiago (Chile) tor, debido al régimen de mezcla al que están sujetos en su interior.

La principal aplicación de la función DTR se encuentra en los modelos de balance de masa, actualmente muy utilizados para el diseño y control de reactores metalúrgicos, siendo importante destacar que el énfasis de este trabajo apunta especialmente a procesos de flotación de minerales.

La incorporación de tales modelos al análisis de la operación de equipos metalúrgicos requiere del conocimiento del régimen de flujo del material en el reactor. Para esto, en principio, es necesario conocer la trayectoria de cada elemento del material que pasa a través del reactor, pero la complejidad 
asociada a esta determinación hace que este método, en la práctica, sea inaplicable. Sin embargo, se puede obtener una buena aproximación al proceso real a través de la distribución de edades de los elementos del material en el flujo de salida, o de la distribución de tiempo de residencia dentro del reactor. Para representar el proceso real se puede ajustar un modelo que prediga la misma distribución de tiempos de residencia o una similar.

En este artículo se realiza una revisión de los principales modelos de DTR aplicables en flotación de minerales y de los aspectos generales relacionados con su determinación experimental. Además, se presentan nuevos modelos para el proceso de flotación continua, que se obtienen como producto de la ponderación de la respuesta semidiscontinua obtenida a escala de laboratorio y la distribución de tiempos de residencia a escala industrial. Los resultados de laboratorio se modelan a través de las expresiones de García-Zúñiga y de Klimpel, y la DTR se representa por el modelo de flujos combinados.

\subsection{Distribución de tiempos de residencia}

Se denomina edad de una partícula al tiempo que transcurre entre su entrada a un estanque o reactor y el instante $t$ de salida de la misma. Obviamente, en un estanque existen partículas de diversas edades, debido a que no todas ellas pasan por él a la misma velocidad ni siguen la misma trayectoria. Es así como se puede definir una función de distribución de edades de salida (1).

Considerando un estanque de volumen activo $V$, a través del cual pasa un material con flujo volumétrico $Q$, y suponiendo que el sistema se encuentra en estado estacionario, el tiempo medio de residencia se obtiene haciendo el siguiente balance:

$$
\left[\begin{array}{c}
\text { Volumen total } \\
\text { de materia en } \\
\text { el estanque a } \\
t=0
\end{array}\right]=
$$

$=\sum_{0}^{\infty}\left[\begin{array}{c}\text { Volumen de material } \\ \text { que entra al } \\ \text { estanque entre } \\ t \mathrm{y} t+\mathrm{d} t\end{array}\right]\left[\begin{array}{c}\text { Fracción de material } \\ \text { que permanece un } \\ \text { tiempo mayor que } t \\ \text { en el estanque }\end{array}\right]$

Matemáticamente :

$$
V=\int_{0}^{\infty} Q \mathrm{~d} t^{\prime}\left[\int_{t^{\prime}}^{\infty} E(t) \mathrm{d} t\right]
$$

donde $E(t)$ es la frecuencia de edades de las partículas que dejan el reactor en el instante $t$, denominada función DTR. Esta tiene unidades de fracción por unidad de tiempo, tal que la fracción de partículas que ingresaron al estanque en $t=0 \mathrm{y}$ lo abandonan entre $t$ y $t+d t$, es $E(t) d t$. Para estanques con comportamiento ideal se cumple que:

$$
\tau=\frac{V}{Q}
$$

donde $\tau$ es el tiempo medio de residencia. Reemplazando en la ec. [2] y desarrollando, se obtiene:

$$
\tau=\int_{0}^{\infty} t E(t) \mathrm{d} t
$$

La dispersión de la distribución del tiempo de residencia está dada por la varianza $\sigma^{2}$, que se define como:

$$
\sigma^{2}=\int_{0}^{\infty}(t-\tau)^{2} E(t) \mathrm{d} t
$$

\subsection{Medición experimental de la DTR}

La función DTR puede determinarse experimentalmente mediante la técnica de estímulo-respuesta. Esta consiste en adicionar un trazador en la alimentación del equipo y medir su concentración en el flujo de salida. Un trazador es una pequeña porción de material de alimentación que ha sido marcado, de tal forma que puede detectarse a la salida del equipo. Diferentes equipos requieren diferentes técnicas experimentales. Dependiendo del sistema, se pueden utilizar trazadores cuya propiedad a medir sea la conductividad, concentración, absorbancia de luz, radiactividad u otras.

El trazador se inyecta en la entrada del reactor en forma de perturbación, tal como: impulso, escalón, sinusoide o simplemente una señal aleatoria. La señal más usada en la práctica por su facilidad de análisis es el impulso.

Entre los factores que deben considerarse para la selección del trazador, se pueden mencionar (2):

- Disponibilidad del trazador y del equipo de detección asociado

- El trazador debe ser detectable de forma precisa, aún en bajas concentraciones, de manera que su introducción no afecte el patrón de flujo de la corriente principal.

- El trazador debe ser miscible y tener propiedades físicas similares a las del flujo principal, cuyo comportamiento de mezcla está bajo investigación. 
- No debe reaccionar químicamente, ni debe absorberse en las paredes u otras partes del equipo.

\subsection{Tipos de modelos de mezcla}

Los modelos de mezcla usualmente empleados en la modelación matemática de reactores metalúrgicos son: el modelo de mezcla perfecta, el modelo de flujo pistón y el modelo de flujos combinados, en el cual se incluyen las anomalías de mezcla que se presentan con mayor frecuencia en la práctica.

\subsubsection{Modelos ideales}

El modelo de mezcla perfecta considera un mezclamiento total con distribución homogénea e instantánea de todas las partículas dentro del equipo o reactor. La concentración de partículas a la salida del reactor, en cualquier instante $t$ considerado, es idéntica a la del interior del equipo en cualquier posición dentro del mismo.

El modelo de flujo pistón considera ausencia total de mezcla en la dirección axial del reactor, produciéndose una secuencia de salida de las partículas en el mismo orden en que ellas entraron al equipo. En este caso, todas las partículas tienen el mismo tiempo de residencia dentro del reactor, $t=\tau$.

Los patrones de flujo observados en la práctica pueden presentar diferentes características en la varianza o desviación estándar de la función DTR, lo cual está asociado con el grado de mezcla existente en el sistema. Los extremos que pueden presentarse son dos: el primero corresponde a una situación de mezcla perfecta, caracterizada porque la varianza de la distribución es $\sigma^{2}=t^{2}$, y el otro extremo asociado a la ausencia total de mezcla en el sistema, caracterizado por $\sigma^{2}=0$.

La expresión matemática que representa la función DTR de los modelos de mezcla perfecta y flujo pistón se obtienen al realizar un balance de masa en un reactor de volumen $V$ que opera en estado estacionario, con un caudal de alimentación constante $Q$ al cual, en el instante $t=0$, se le aplica trazador como un impulso, tipo función delta de Dirac, para evaluar la DTR del trazador en la salida. Así, se obtiene:

$$
E(t)=\frac{1}{\tau} e^{-t / \tau}
$$

para flujo con mezcla perfecta, y

$$
E(t)=\delta(t-\tau)
$$

para un reactor con régimen de mezcla tipo flujo pistón.

\subsubsection{Modelo de mezcladores en serie perfectamente mezclados}

Un método para establecer una descripción empírica de una distribución de tiempos de residencia, es considerar la función DTR originada por un número de reactores en serie perfectamente mezclados. Para determinar el número equivalente de reactores incluidos en el modelo teórico, se compara la curva DTR experimental con la obtenida considerando $N$ reactores iguales en serie y perfectamente mezclados. Si existe gran diferencia entre ambas curvas, se modifica el número de reactores, hasta lograr el mejor ajuste posible. Así, considerando $N$ reactores en serie, cada uno con función DTR de $E_{1}(t), E_{2}(t), E_{3}(t), \ldots, E_{\mathrm{N}}(t)$ y tiempos medios de residencia $\tau_{1}, \tau_{2}, \tau_{3}, \ldots, \tau_{\mathrm{N}}$, respectivamente, la expresión que representa la distribución de tiempo de residencia del sistema global es:

$E(t)=\sum_{\mathrm{i}=1}^{\mathrm{N}} \frac{\tau_{\mathrm{i}}^{(\mathrm{N}-2)} \exp \left(-t / \tau_{\mathrm{i}}\right)}{\left(\tau_{\mathrm{i}-} \tau_{1}\right)\left(\tau_{\mathrm{i}-} \tau_{2}\right) \ldots\left(\tau_{\mathrm{i}-} \tau_{\mathrm{k}}\right) \ldots\left(\tau_{\mathrm{i}-} \tau_{\mathrm{N}}\right)}$

$\operatorname{con} j \neq k$ y $\tau_{\mathrm{j}} \neq \tau_{\mathrm{k}}$. La ec. [8] no puede usarse para $N$ reactores iguales perfectamente mezclados en serie, es decir, $\tau_{1}=\tau_{2}=\tau_{3}=\ldots=\tau / N$, ya que para este caso se obtiene:

$$
E(t)=\frac{N^{\mathrm{N}}(t / \tau)^{\mathrm{N}-1} \exp (-N t / \tau)}{\tau(N-1) !}
$$

El problema de caracterizar los resultados experimentales se reduce, entonces, a determinar los parámetros $N$ y $\tau$ asociados al transporte de material a través del reactor a partir de los momentos de primer y segundo orden de la distribución $E(t)$, es decir, de la media y la varianza.

\subsubsection{Anomalías de mezcla en sistemas reales}

- Zonas muertas: Corresponden a regiones detenidas dentro del equipo o a zonas de muy poco contacto con el fluido en movimiento. La permanencia de las partículas en esta zona es bastante más prolongada que el tiempo medio de residencia del conjunto (en muchos casos reales 2 a 3 veces superior a $\tau$ ).

- Cortocircuito: Existen zonas preferenciales en el equipo (canalizaciones), que son aprovechadas por ciertas partículas para cortocircuitar el reactor, dirigiéndose directamente al flujo de salida. 
En términos prácticos, algunos autores consideran que todas aquellas partículas cuyo tiempo de residencia es menor a $0,1 \tau$, habrán cortocircuitado la zona activa del reactor.

- Recirculación interna: Se denomina recirculación interna o reciclaje a cierta proporción del flujo de salida que regresa o se recicla a la entrada del reactor, disminuyendo, por tanto, el volumen activo del equipo.

\subsubsection{Modelo de flujos combinados}

El modelo de flujos combinados o mixtos fue sugerido por Cholette y Cloutier (3), quienes consideraron que un patrón de flujo debe incluir etapas de mezcla perfecta y flujo pistón, efectos de espacio muerto, cortocircuitos, retardo en la respuesta del reactor frente a perturbaciones externas, o cualquier combinación posible de éstos. El modelo combinado general, para $\theta=t / \tau$, se presenta a continuación:

$$
E(\theta)=\left\{\begin{array}{cc}
0 & \theta<\epsilon / \tau \\
\frac{(N \eta)^{\mathrm{N}}(\theta-\epsilon / \tau)^{\mathrm{N}-1} \exp [-N \eta(\theta-\epsilon / \tau)]}{(N-1) !} & \theta \geq \epsilon / \tau
\end{array}\right.
$$

Se puede observar que si $\eta=1$ y $\epsilon / \tau=0$, se obtiene el conocido modelo de $N$ reactores iguales perfectamente mezclados.

El parámetro $\eta$ en la ec. [10] mide la eficiencia de mezcla existente dentro de cada reactor. Para el caso de mezcla perfecta, $\eta=1$, mientras que para flujo pistón (que implica ausencia total de mezcla en la celda), $\eta \rightarrow \infty$. La presencia de espacios muertos en el sistema origina valores de $\eta>1$, mientras que el efecto de cortocircuito resulta en valores de $\eta<1$. De esta forma, cualquier desviación de $\eta$ con respecto a la unidad indica que no existe mezcla perfecta en el volumen completo de cada reactor.

El parámetro $\epsilon / \tau$ de la ec. [10] mide el tiempo de retardo del sistema en responder frente a perturbaciones externas. Si $\epsilon / \tau>0$, el sistema responde con retraso respecto al caso ideal de mezcla perfecta, siendo $\epsilon / \tau=0$ para el caso de mezcla perfecta, y $\epsilon / \tau=1$ para el caso extremo de flujo pistón. Si existe cortocircuito sucede lo inverso, obteniéndose una respuesta anticipada del sistema, con lo que $\epsilon / \tau$ toma un valor negativo, $\epsilon / \tau<0$.

Los valores de los parámetros $\eta$ y $\epsilon / \tau$, en cada caso particular, pueden calcularse a través del método de los momentos, esto es, a través de la media y la varianza de las distribuciones $E(t)$ y $E(\theta)$, o bien mediante técnicas apropiadas de regresión no lineal múltiple.

\section{FLOTACIÓN EN LABORATORIO FRENTE A PLANTA INDUSTRIAL}

Es importante precisar algunas de las principales diferencias entre la escala industrial y la de laboratorio, debido a que éstas son las responsables de las discrepancias que se presentan entre los valores de la constante cinética y de los tiempos con que se obtiene una misma recuperación en ambos sistemas:

- El sistema de remoción de productos es absolutamente distinto en ambos procesos, ya que para el caso de laboratorio se altera de forma diferenciada tanto la altura de la columna de espuma como la hidrodinámica de la parte inmediatamente inferior a ésta. La formación de la fase espuma es de tipo discontinuo y su extracción de tipo pulsante.

- La distancia recorrida por las burbujas mineralizadas en el laboratorio es comparativamente menor que en la situación industrial, disminuyendo la probabilidad de destrucción de la unidad burbuja-partícula.

- La hidrodinámica y la distribución de tamaños de burbujas es diferente en ambas escalas. En sistemas discontinuos, la turbulencia es mayor en la interfase pulpa/espuma.

- El régimen de mezcla en las celdas de flotación es diferente entre el laboratorio y la planta.

- La relación agua/sólido y el nivel de pulpa en la flotación discontinua no son constantes, por tratarse de un sistema que incluye remoción de concentrados sin adición extra de pulpa.

- En el sistema discontinuo todas las partículas tienen un tiempo de residencia reducido a un rango estrecho, mientras que en el caso continuo, existe una distribución de tiempos de residencia.

En resumen, se puede concluir que resulta inevitable obtener diferencias en los resultados metalúrgicos generados en ambas escalas y que, en general, las condiciones del laboratorio se acercan más al ideal que las que imperan en la planta industrial. Por lo tanto, la respuesta metalúrgica a escala de laboratorio es más favorable que la que se obtiene en los procesos continuos.

\section{UTILIZACIÓN DE LA DTR EN EL ESCALAMIENTO DE RESULTADOS DE FLOTACIÓN SEMICONTINUA}

Gutiérrez y col. (4) desarrollaron en 1987 una metodología para el análisis del régimen de mezcla de bancos de flotación para la derivación de la cinética de flotación industrial a partir de los respectivos modelos de flotación semicontinua. 
Estos autores modelan la mezcla en la celda de flotación considerando que una parte del flujo que entra al sistema cortocircuita en el interior de éste, apareciendo directamente en el flujo del relave, sin mezclarse. Como anomalía a la mezcla perfecta, considera que en la celda existe un volumen muerto. La parte restante del flujo es la fracción activa de la celda, que se comporta en parte en régimen de flujo pistón y en parte en régimen de mezcla perfecta.

Este enfoque conduce al concepto de DTR efectiva, definida por una curva que combina la DTR ideal con la experimental y que se divide en tres zonas para su cuantificación. Entre 0 y $\theta_{1}$, el escalamiento se efectúa a través de la DTR ideal y $\theta_{1}$ representa el valor de $\theta$ para el cual las partículas en cortocircuito salen del banco. Así, en esta zona se debe calcular:

$$
\int_{0}^{\theta_{1}} r_{\mathrm{B}}(\theta) E(\theta)^{\mathrm{id}} \mathrm{d} \theta
$$

donde $r_{\mathrm{B}}(\theta)$ es la recuperación semicontinua representada por los modelos de García-Zúñiga, Klimpel u otros, y $E(\theta)^{\text {id }}$ es la distribución de tiempos de residencia ideal, que corresponde al modelo de $N$ reactores perfectamente mezclados y conectados en serie. Se hace notar que en estas condiciones la integral tiene solución analítica.

Por su parte, en la zona 2 , entre $\theta_{1}$ y $\theta_{3}$, el escalamiento se realiza a través de la DTR experimental y $\theta_{3}$ representa el valor de $\theta$ para una de las intersecciones entre las curvas experimentales e ideal, la más alejada del origen, debiéndose calcular en esta zona la siguiente integral:

$$
\int_{\theta_{1}}^{\theta_{3}} r_{\mathrm{B}}(\theta) E(\theta)^{\mathrm{ex}} \mathrm{d} \theta
$$

donde $r_{\mathrm{B}}(\theta)$ tiene el mismo significado que en la zona 1, y $E(\theta)^{\text {ex }}$ corresponde a la distribución de tiempo de residencia real de las partículas en el interior del reactor. Esta respuesta puede modelarse a través del modelo de flujos combinados. Una solución analítica para esta integral resulta compleja, razón por la cual se recurre al empleo de métodos numéricos para lograr su cuantificación.

En la zona 3 se efectúa el escalamiento de forma análoga a la zona 1, y es posible resolver analíticamente la siguiente integral:

$$
\int_{\theta_{3}}^{\infty} r_{\mathrm{B}}(\theta) E(\theta)^{\mathrm{id}} \mathrm{d} \theta
$$

Por último, la recuperación efectiva corresponde a la suma de las integrales que definen cada una de las zonas mencionadas.
Aunque Gutiérrez proporciona una herramienta de utilidad para la interpretación de las características de mezcla en reactores continuos, el hecho de atribuir una interpretación física invariante a los desplazamientos en tiempo y altura de la función DTR introduce a una incertidumbre que puede llevar a conclusiones erróneas con respecto al régimen de mezcla. En efecto, dichos desplazamientos de la DTR experimental respecto a la ideal se pueden deber a un cortocircuito, una recirculación o a una combinación de ambas -si el desplazamiento es en el sentido negativo del eje correspondiente al tiempoy no a un solo efecto como se presupone en el modelo. Igual ambigüedad ocurre en la interpretación de los desplazamientos en el sentido positivo del eje del tiempo y, en general, de cambios en la altura y varianza de la DTR.

\section{ESCALAMIENTO A TRAVÉS DEL MODELO DE $N$ REACTORES IGUALES PERFECTAMENTE MEZCLADOS}

Como se vio anteriormente, el escalamiento de resultados de flotación obtenidos en laboratorio a planta industrial se puede efectuar a través de la expresión:

$$
r=\int_{0}^{\infty} r_{\mathrm{B}}(\theta) E(\theta) \mathrm{d} \theta
$$

donde $r_{\mathrm{B}}(\theta)$ es la recuperación obtenida a escala de laboratorio y $E(\theta)$ la función DTR adimensionalizada que caracteriza la operación industrial. Utilizando la función de transferencia correspondiente al modelo de $N$ reactores iguales perfectamente mezclados y conectados en serie (ec. [9]) para representar $N$ celdas de flotación, se obtienen los modelos clásicos de flotación en continuo. Utilizando el modelo de García-Zúñiga para representar la recuperación semicontinua, a escala laboratorio, es decir:

$$
r_{\mathrm{B}}(\theta)=R[1-\exp (-k \tau \theta)]
$$

donde $R$ es la recuperación máxima lograda, en $\%, k$ la constante cinética de flotación de la especie de interés, con unidades $1 / \mathrm{min}$, y $\theta$ el tiempo adimensional de flotación, $t / \tau$. Al integrar la ec. [14], se obtiene:

$$
r=R\left[1-\left(\frac{N}{N+k \tau}\right)^{\mathrm{N}}\right]
$$

así, también, si la recuperación semicontinua se modela a través del modelo de Klimpel, 


$$
r_{\mathrm{B}}(\theta)=R\left[1-\left(\frac{1}{k \tau \theta}\right)(1-\exp [-k \tau \theta])\right]
$$

con las mismas consideraciones anteriores, se obtiene:

$$
r=R\left[1-\frac{1-N^{N-1}(N+k \tau)^{1-\mathrm{N}}}{(N-1) k \tau / N}\right]
$$

Estos modelos ofrecen sólo una aproximación de la situación real en continuo, debido a que se ha comprobado experimentalmente que la distribución de tiempos de residencia en el proceso de flotación puede alejarse significativamente de la predicción del modelo de los $N$ reactores iguales perfectamente mezclados y conectados en serie.

\section{ESCALAMIENTO A TRAVÉS DEL MODELO DE FLUJOS COMBINADOS}

De acuerdo con lo discutido anteriormente, parece más razonable integrar directamente la expresión del modelo de flujos combinados modelando la recuperación a través de alguno de los modelos cinéticos más comunes, como García-Zúñiga o Klimpel, sin hacer mayores suposiciones acerca del complejo comportamiento hidrodinámico del sistema que, como se dijo, obligan a definir una DTR efectiva y consecuentemente tres zonas sobre las cuales se realizará la integración con diferentes valores para la función $E(\theta)$ según sea ésta. Por lo anterior, el enfoque planteado en el presente trabajo constituye una aproximación más objetiva del problema.

\subsection{Modelo de García-Zúñiga para la recuperación}

Si se asume el modelo de García-Zúñiga para la recuperación semicontinua a escala laboratorio, ec. [15], reemplazándola en la ec. [14], y considerando la distribución de tiempos de residencia dada por la ec. [10], se obtiene:

$$
r=\int_{0}^{\epsilon / \tau} r_{\mathrm{B}}(\theta) E(\theta) \mathrm{d} \theta+\int_{\epsilon / \tau}^{\infty} r_{\mathrm{B}}(\theta) E(\theta) \mathrm{d} \theta
$$

De acuerdo con la definición de $E(\theta)$, la primera integral es 0. Así, el problema se reduce a resolver la segunda integral. Para el caso en que $N$ es un valor par, reemplazando la ec. [10] y reordenando, se llega a la siguiente expresión:

$$
\begin{gathered}
r=\frac{R(N \eta)^{\mathrm{N}} \exp (N \eta)\left(\frac{\epsilon}{\tau}\right)}{(N-1) !} \\
{\left[\int_{\epsilon=\tau}^{\infty}\left(\theta-\left(\frac{\epsilon}{\tau}\right)\right]^{\mathrm{N}-1} \exp (-N \eta \theta) \mathrm{d} \theta-\right.} \\
\left.\int_{\epsilon / \tau}^{\infty}\left(\theta-\left(\frac{\epsilon}{\tau}\right)\right]^{\mathrm{N}-1} \exp (-(N \eta+k \tau) \theta) \mathrm{d} \theta\right]
\end{gathered}
$$

Utilizando el teorema del binomio de Newton [5], se puede plantear la siguiente igualdad,

$$
\left[\left(-\left(\frac{\epsilon}{\tau}\right)+\theta\right]^{N-1}=\sum_{j-1}^{N} \frac{(N-1) !\left(\frac{\epsilon}{\tau}\right)^{N-j}}{(j-1) !(N-j) !} \theta^{j-1}\right.
$$

Reemplazando en la ec. [20] y desarrollando, se obtiene:

$$
\begin{aligned}
& r=R(N \eta)^{\mathrm{N}} \exp (N \eta)\left(\frac{\epsilon}{\tau}\right) \sum_{\mathrm{j}=1}^{\mathrm{N}} \frac{\left(-\frac{\epsilon}{\tau}\right)^{N-\mathrm{j}}}{(j-1) !(N-j) !} \\
& {\left[\int_{\epsilon \in \tau}^{\infty} \theta^{\mathrm{j}-1} \exp (-N \eta \theta) \mathrm{d} \theta-\right.} \\
&\left.-\int_{\epsilon / \tau}^{\infty} \theta^{\mathrm{j}-1} \exp (-(N \eta+k \tau) \theta) \mathrm{d} \theta\right]
\end{aligned}
$$

La solución general de las integrales de la ec. [22] viene dada por:

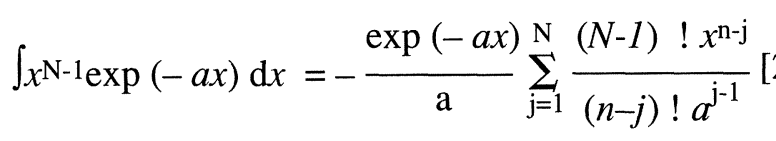

Considerando esta solución y resolviendo, se obtiene:

$$
\begin{gathered}
r=R\left[\sum_{\mathrm{j}=1}^{\mathrm{N}} \sum_{\mathrm{i}=1}^{\mathrm{j}} \frac{(-1)^{\mathrm{j}}(\epsilon / \tau)^{\mathrm{N}-\mathrm{i}}(N \eta)^{\mathrm{N}-\mathrm{i}}}{(N-j) !(j-i) !}\right]- \\
-R\left[\frac{(N \eta)^{\mathrm{N}} \exp [-k \tau(\epsilon / \tau)] \sum_{\mathrm{j}=1}^{\mathrm{N}} \sum_{\mathrm{i}=}^{\mathrm{j}}}{(-1)^{\mathrm{j}}(\epsilon / \tau)^{\mathrm{N}-\mathrm{i}}}\right. \\
\\
(N-j) !(j-i) !(N \eta+k \tau)^{\hat{\mathrm{i}}}
\end{gathered}
$$

Por inducción matemática, se puede demostrar que la primera doble sumatoria de la ec. [24] es igual a 1, por lo que esta ecuación se reduce a: 


$$
\begin{array}{r}
r=R\left[1-(N \eta)^{\mathrm{N}} \exp [-k \tau(\epsilon / \tau)]\right. \\
\left.\sum_{\mathrm{j}=1}^{\mathrm{N}} \sum_{\mathrm{i}=1}^{\mathrm{j}} \frac{(-1)^{\mathrm{j}}(\epsilon / \tau)^{\mathrm{N}-\mathrm{i}}}{(N-j) !(j-i) !(N \eta+k \tau)^{\hat{1}}}\right]
\end{array}
$$

Esta expresión representa la solución general del escalamiento de resultados semicontinuos de flotación hasta un nivel industrial, representando la recuperación de laboratorio por el modelo de GarcíaZúñiga y la DTR de planta según el modelo de flujos combinados. Los parámetros a determinar a escala de laboratorio son la recuperación máxima, $R$, y la constante cinética de flotación de la especie de interés, $k$, mientras que $\eta$ y $\epsilon / \tau$ se determinan a partir de los valores experimentales de la DTR obtenidos a escala industrial. $N$ es el número de celdas del banco y $\tau$ el tiempo medio de residencia en el banco completo. Si $N$ es impar, el exponente $j$ de (1) en la ec. [25] se debe reemplazar por $j+1$.

\subsection{Modelo de Klimpel para la recuperación}

Para determinar la solución general de escalamiento, utilizando el modelo de Klimpel para representar la recuperación a escala laboratorio (ec. [17]) en lugar del de García-Zúñiga, se debe realizar un análisis análogo al anterior, obteniéndose la siguiente expresión analítica para $N$ par:

$$
\begin{gathered}
r=R\left[1-\frac{1}{k \tau(\epsilon / \tau)} \sum_{\mathrm{j}=2}^{\mathrm{N}} \sum_{\mathrm{i}=1}^{\mathrm{j}}\right. \\
\frac{(-1) \mathrm{j}(j-i)(\epsilon / \tau)^{\mathrm{N}-\mathrm{i}}(N \eta)^{\mathrm{N}-\mathrm{i}}}{(j-1)(j-i) !(N-j) !}+\frac{(N \eta)^{\mathrm{N}} \exp [-k \tau(\epsilon / \tau)]}{k \tau(\epsilon / \tau)} \\
\left.\sum_{\mathrm{j}=2}^{\mathrm{N}} \sum_{\mathrm{i}}^{\mathrm{j}} \frac{(-1)^{\mathrm{j}}(j-i)(\epsilon / \tau)^{\mathrm{N}-\mathrm{i}}}{(j-1)(N-j) !(j-\mathrm{i}) !(N \eta+k \tau)^{\hat{1}}}\right]
\end{gathered}
$$

Para $N$ impar, el exponente $j$ de (-1) en la ec. [26] se debe reemplazar por $j+1$.

\section{COMPARACIÓN DE METODOLOGÍAS DE ESCALAMIENTO DE RESULTADOS DE LABORATORIO A PLANTA INDUSTRIAL}

En la figura 1 se muestra una curva de DTR experimental obtenida en un banco de flotación

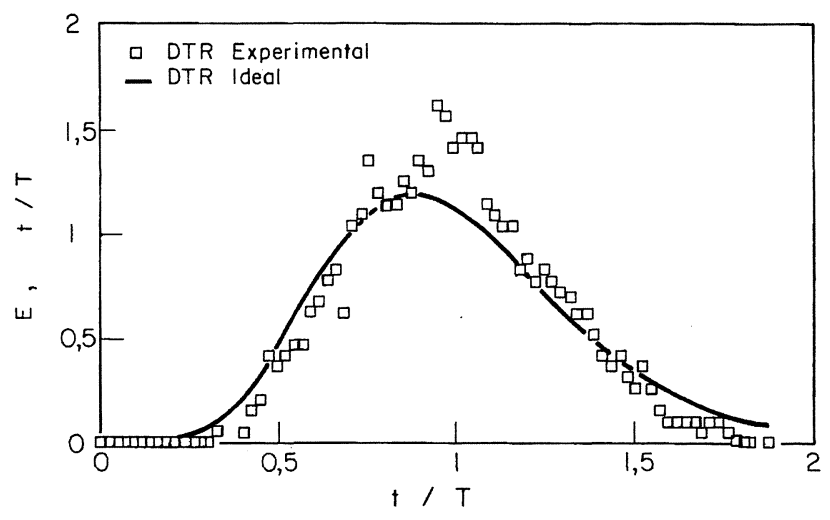

FIG. 1.- Curva DTR experimental de un banco de flotación de ocho celdas con $\tau=21,5 \mathrm{~min}$. Se muestra la curva ideal obtenida con la ec. [9] para $N=8$.

FIG. 1.-Experimental RTD curve with $\tau=21.5 \mathrm{~min}$ for an 8-cell flotation bank. The ideal curve obtained with eq. [9] for $N=8$ is included.

industrial formado por ocho celdas con un tiempo promedio de residencia de 21,5 min. A modo de comparación, en la misma figura se muestra la curva generada al resolver el modelo de $N$ reactores en serie perfectamente mezclados, con $N=8$. Sin embargo, es necesario dejar constancia de que el valor de $N$, al usar el modelo ideal, se varía hasta lograr un mejor ajuste asumiendo que no existen mezcladores perfectos en la realidad, razón por la cual, en este caso, la comparación se efectúa en la condición más adversa para el modelo de los $N$ reactores perfectamente mezclados. Es por esta razón que se observa una marcada diferencia entre las curvas experimental y ajustada; no obstante, es necesario reconocer que aunque se varíe $N$ el ajuste continuará siendo de calidad insuficiente, porque el modelo de $N$ mezcladores perfectos y conectados en serie sólo constituye una visión idealizada de la realidad.

Procede decir que el modelo de flujos combinados permite acceder a un mayor acercamiento con la distribución de tiempo de residencia de las partículas en un banco de celdas industriales, y que, por lo tanto, dicho modelo reproducirá con mayor precisión la DTR del banco.

De acuerdo con lo expresado anteriormente, es posible afirmar que la ec. [14] que define el escalamiento laboratorio-planta, al utilizar el modelo de flujos combinados para ajustar la DTR experimental, permitirá obtener una respuesta metalúrgica, caracterizada por la recuperación y la ley de los elementos valiosos, que se ajustará mejor a la realidad.

En la figura 2 se muestran dos curvas de cinética de flotación industrial, obtenidas a partir de la curva experimental de DTR en planta y de pruebas de flotación a escala de laboratorio, cuyos resultados se 


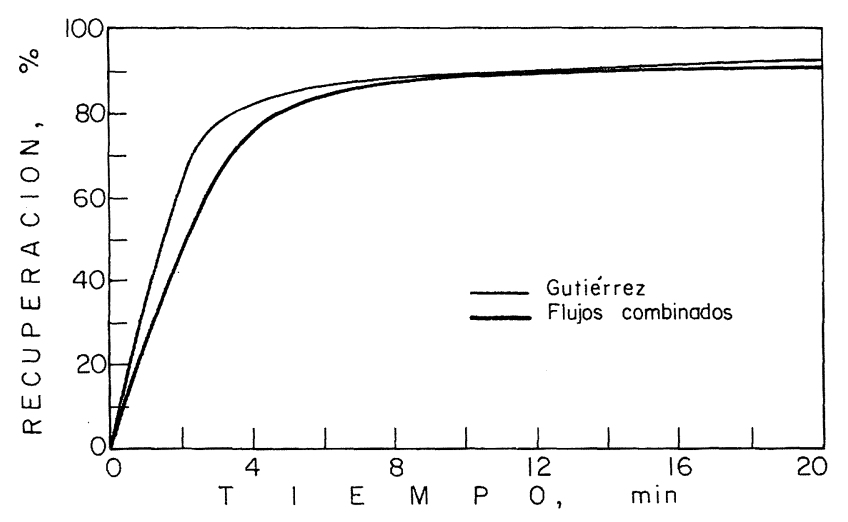

FIG. 2.- Predicción de la cinética de flotación industrial según el modelo de Gutiérrez y el de flujos combinados, usando la ecuación de Klimpel para la recuperación.

FIG. 2.- Prediction of industrial flotation kinetics according to the Gutiérrez'model and the combined-flow model. Klimpel equation for the recovery is employed in both cases.

ajustaron con el modelo de Klimpel. Por otra parte, las curvas cinéticas industriales se obtuvieron a través de los métodos de Gutiérrez y del que se presenta en este trabajo.

Al comparar ambas curvas, queda al descubierto una diferencia apreciable a tiempos cortos, lo que en términos prácticos significa que el modelo de Gutiérrez es más optimista en sus predicciones a dichos tiempos que el modelo que se presenta en esta publicación. Esto tiene incidencia para tiempos de flotación antes de alcanzar el "hombro" en la curva de recuperación $v s$. tiempo; sin embargo, para tiempos mayores ambos enfoques son convergentes.

Un aspecto que no se ha abordado con el énfasis que requiere, es que el modelo propuesto por los autores elimina el análisis de caja negra subyacente a los métodos numéricos $\mathrm{y}$, por lo tanto, permite analizar el problema del escalamiento desde una perspectiva analítica más cercana a la realidad, si se considera que utiliza el modelo de flujos combinados para modelar la función $E(\theta)$ e integrar la ec. [14] para escalamiento.

La expresión analítica propuesta permite, además, evaluar los eventuales cambios que experimentan los parámetros con las condiciones de operación $\mathrm{y}$, consecuentemente, el efecto que tendrán dichos cambios en la respuesta metalúrgica, caracterizada por la recuperación y la ley de los elementos valiosos, que se desea caracterizar.

\section{CONCLUSIONES}

- Se han desarrollado expresiones analíticas para el escalamiento de resultados desde el laboratorio a la planta industrial, que permiten dar cuenta de la recuperación en el proceso continuo a partir de ensayos semicontinuos a escala de laboratorio y de la DTR del sistema industrial.

- El modelo desarrollado constituye una poderosa herramienta de simulación de proceso, si se considera que los parámetros $\eta$ y $\epsilon / \tau$ son susceptibles de ser correlacionados con las condiciones de operación industrial.

- El modelo propuesto en esta publicación permite acceder a un mayor acercamiento a la realidad que define la dinámica de los procesos continuos, si se compara con aquellos que utilizan el modelo de $\operatorname{los} N$ reactores perfectamente mezclados y conectados en serie para caracterizar la DTR, o que definen una DTR efectiva cuya definición depende de la perspectiva desde la cual se haga el análisis.

\section{Agradecimiento}

Los autores agradecen a la Universidad de Santiago de Chile y al Centro de Investigación Minera y Metalúrgica de Chile (CIMM) la oportunidad brindada para desarrollar este trabajo.

\section{REFERENCIAS}

(1) Ruiz, M., Magne, L. y Menacho, J. Distribución de Tiempos de Residencia, Serie Monografías en Conminución, núm. 6. Proyecto Desarrollo Tecnológico de la Conminución en la Industria Nacional. Santiago (Chile), junio de 1992.

(2) Wen, C.Y. y FAN, L.T. Models for Flow Systems and Chemical Reactors. Ed. Marcel Dekker, Inc. Nueva York, 1975.

(3) Cholette, A. y Cloutier, L. Can. J. Chem. Eng., 37, 1959: 105.

(4) GutiérRez, L. Revisión de Modelación Matemática en Flotación de Minerales. Seminario Desarrollo y Perspectiva de la Metalurgia Extractiva. Universidad de Santiago de Chile, 1991.

(5) Larsen, H.: Mathematical Tables, Formulas and Curves. Edición ampliada. Michigan, 1953. 